\title{
Transfer matrix method of linear multibody systems for free vibration analysis of beam carrying elastically mounted point masses
}

\author{
Laith K. Abbas ${ }^{1, a *}$, Dongyang Chen ${ }^{1, b}$, Guoping Wang ${ }^{1, c}$ and Xiaoting Rui ${ }^{1, d}$ \\ ${ }^{1}$ Institute of Launch Dynamics, Nanjing University of Science and Technology, Nanjing, 210094, \\ China \\ a laithabbass@yahoo.com , bccdy_1988@sina.cn , cwgp1976@163.com , druixt@163.net
}

\begin{abstract}
Keywords: Transfer matrix method, Spring-mass, Recursive eigenvalue search, Free vibration.
\end{abstract}
\begin{abstract}
The analysis of natural vibration characteristics has become one of the important steps of the manufacture and dynamic design in different industries. An efficient approach called transfer matrix method of linear multibody systems (MSTMM) is proposed to determine the free vibration of a cantilever beam carrying any number of elastically mounted point masses vibrating in a plane. The components of the transfer matrix are all functions of the system's natural frequency. The overall transfer equation only involves boundary state vectors, whereas the state vectors at all other connection points do not appear. The state vectors at the boundary are composed of displacements, rotation angles, bending moments and shear forces, which are partly known and partly unknown. Recursive eigenvalue search algorithm is used to determine the system frequencies. A numerical example is presented to demonstrate the validity of the proposal method. The proposed method is easy to formulate, systematic to apply, and simple to code.
\end{abstract}

\section{Introduction}

The vibration problem of beam-type structures is of particular urgent issue in many branches of modern aerospace, mechanical, and civil engineering. Natural vibration frequencies and modes are one of the most important dynamic characteristics of these kinds of systems. For example, the precision in manufacturing can be highly influenced by vibrations. If the vibration characteristics cannot be solved or pre-estimated exactly when designing a mechanism system, it is often hard to obtain a good dynamic performance of the mechanism system and consequently hard to control its vibration. Recently, there is a vast number of articles in the published literature which are concerned with the computation of the eigenvalues problems of Euler-Bernoulli beams, subjected to different boundary conditions and carrying any number of concentrated elements (such as elastically mounted point masses, rigidly attached point masses, translational springs, and/or rotational springs) [1-5].

The transfer matrix method of linear multibody systems (MSTMM) has been developed for a long time and has been used widely in structural analysis of linear and nonlinear systems. Applications of MSTMM range from vibration analysis, modeling of composite structures and multibody systems for computing static deformations, natural vibrations, steady state and transient dynamical response, to damage identification [6-8]. The general idea of MSTMM is to firstly break up a complicated system into elements with simple dynamic properties, which can be readily expressed in matrix form and treated in the same manner (for each element, it is possible to obtain the close form expression of the transfer matrix giving the displacements and the forces applied to one extremity to the displacements and forces applied to the other extremity). In other words, on an element level, the governing partial and ordinary differential or algebraic equations are transformed to algebraic transfer equations, where the output state results from a product of the input state and an element specific transfer matrix. These component matrices are considered as building blocks. In fact, the transfer matrix of such components does not need to be re-deduced, but may be taken directly from a transfer matrix library provided by Ref. [7]. These element transfer matrices are then assembled according to the topology of the flexible multibody system, ending up with a system of linear algebraic equations called the overall transfer equation. After eliminating the boundary conditions, the overall transfer matrix needs to be singular, which is checked by its determinant to be zero. Recursive eigenvalue search algorithm [9] is used to 
determine the system Eigen frequencies.

The proposed scenario in this paper is concerned essentially with the same mechanical system described in Ref. [5] but here the natural frequencies of cantilever beam carrying any number of elastically mounted point masses vibrating in a plane are investigated using MSTMM.

\section{Problem Statement and Formulation of Eigenvalue Problem Using MSTMM}

The problem to be under study in this paper is the bending vibration problem of the system shown in Fig. 1. The system composed of continues elements and discrete elements vibrating in a plane. It consists of a cantilever Euler-Bernoulli beam carrying triple spring-mass systems across the span [5]. The length, mass per unit length, location of the spring-mass attachment point, bending rigidity, spring stiffness and lumped mass are $L, \bar{m}, x_{i}, E I, K_{y_{i}}$ and $M_{i}$, respectively, $(i=1,3)$. The transfer equation between the input end and output end of any component in the system, for example, beam, spring, lumped mass can be stated as $\boldsymbol{Z}_{O}=\boldsymbol{U} \boldsymbol{Z}_{I}$, where $\boldsymbol{Z}$ is the state vector and $\boldsymbol{U}$ is the component transfer matrix. For the system vibrating in a plane, $Z$ consists the state variables (SVs) in the global coordinates including displacement $Y$, cross section small rotation angle $\Theta_{z}$, bending moment $M_{z}$ and shear force $Q_{y}$ [7]. The spring, lumped mass and beam transfer equations and matrices are respectively [6-9]

$$
\begin{aligned}
& \boldsymbol{Z}_{O}=\boldsymbol{U}^{\text {spring }} \boldsymbol{Z}_{I} \rightarrow\left[\begin{array}{c}
Y \\
Q_{y}
\end{array}\right]_{O}=\left[\begin{array}{ccc}
1 & -1 / k \\
0 & 1
\end{array}\right]\left[\begin{array}{c}
Y \\
Q_{y}
\end{array}\right]_{I}, \boldsymbol{Z}_{O}=\boldsymbol{U}^{\text {mass }} \boldsymbol{Z}_{I} \rightarrow\left[\begin{array}{c}
Y \\
Q_{y}
\end{array}\right]_{O}=\left[\begin{array}{cc}
1 & 0 \\
m \omega^{2} & 1
\end{array}\right]\left[\begin{array}{c}
Y \\
Q_{y}
\end{array}\right]_{I}, \\
& \boldsymbol{Z}_{O}=\boldsymbol{U}^{\text {beam }} \boldsymbol{Z}_{I} \rightarrow\left[\begin{array}{c}
Y \\
\Theta_{z} \\
M_{z} \\
Q_{y}
\end{array}\right]_{O}=\left[\begin{array}{ccccc}
a_{1} & a_{2} & a_{3} & a_{4} \\
a_{5} & a_{6} & a_{7} & a_{8} \\
a_{9} & a_{11} & a_{11} & a_{12} \\
a_{13} & a_{14} & a_{15} & a_{16}
\end{array}\right]\left[\begin{array}{c}
Y \\
\Theta_{z} \\
M_{z} \\
Q_{y}
\end{array}\right]_{I} . \\
& a_{1}=S(\lambda l), a_{2}=T(\lambda l) / \lambda, a_{3}=G(\lambda l) / E I \lambda^{2}, a_{4}=V(\lambda l) / E I \lambda^{3}, a_{5}=\lambda V(\lambda l), a_{6}=S(\lambda l), \\
& a_{7}=T(\lambda l) / E I \lambda, a_{8}=G(\lambda l) / E I \lambda^{2}, a_{9}=E I \lambda^{2} G(\lambda l), a_{10}=E I \lambda V(\lambda l), a_{11}=S(\lambda l), a_{12}=T(\lambda l) / \lambda, \\
& a_{13}=E I \lambda^{3} T(\lambda l), a_{14}=E I \lambda^{2} G(\lambda l), a_{15}=\lambda V(\lambda l), a_{16}=S(\lambda l), S=\frac{c h+c}{2}, T=\frac{s h+s}{2}, G=\frac{c h-c}{2}, \\
& V=\frac{s h-s}{2}, c h=\cosh \lambda l, s h=\sinh \lambda l, c=\cos \lambda l, s=\sin \lambda l .
\end{aligned}
$$

where $m=\left\langle M_{1}\right.$ or $M_{2}$ or $\left.M_{3}\right\rangle, k=\left\langle K_{y_{1}}\right.$ or $K_{y_{2}}$ or $\left.K_{y_{3}}\right\rangle, \lambda=\sqrt[4]{\bar{m} \omega^{2} / E I}$ is the eigenvalue, $l$ is the beam segment length and $\omega$ is the natural vibration frequency. A systematic MSTMM procedure is proposed which can be used to deal with the vibration problem of the structure shown in Fig.1. In MSTMM, there are different topologies of dynamics models such as chain, tree and closed-loop systems. In the context of MSTMM, the SVs and transfer directions of the complex structure shown in Fig. 1(a) are demonstrated in Fig. 1(b). The transfer directions of a system are always from the tip(s) to the root. In Fig. 1(b), the vibrating system is a tree topology in the MSTMM algorithm and

a)

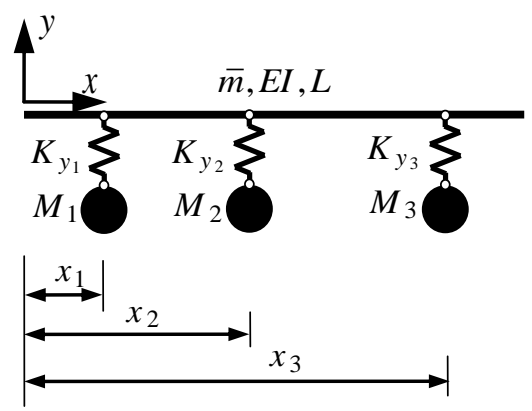

b)

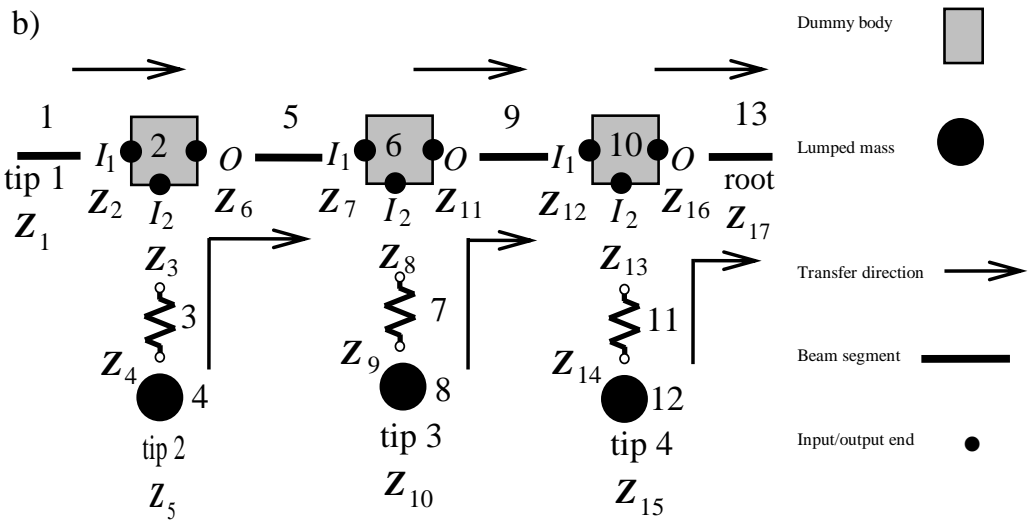

Figure 1. (a) Cantilever beam carrying three spring-mass system, and (b) as well as corresponding elements 
comprised of 13 components. Dummy rigid body (massless rigid body) components 2, 6 and 10 are branches. It has 2 input ends and 1 output end. Components 1, 5, 9 and 13 are beam segments. Spring components are 3,7 and 11. Lumped mass components are 4, 8 and 12. The entire system has totally 5 boundaries $\left(n_{b}=5\right)$, namely, $\boldsymbol{Z}_{17}, \boldsymbol{Z}_{1}, \boldsymbol{Z}_{5}, \boldsymbol{Z}_{10}$ and $\boldsymbol{Z}_{15}$ at the root, tips $1,2,3$ and 4 , respectively. Following the MSTMM tree algorithm [8], the main transfer equation (TE) of the system is

$$
\begin{aligned}
& \boldsymbol{Z}_{17}=\boldsymbol{T}_{1-17} \boldsymbol{Z}_{1}+\boldsymbol{T}_{5-17} \boldsymbol{Z}_{5}+\boldsymbol{T}_{10-17} \boldsymbol{Z}_{10}+\boldsymbol{T}_{15-17} \boldsymbol{Z}_{15} . \\
& \text { where } \boldsymbol{T}_{1-17}=\boldsymbol{U}_{13} \boldsymbol{U}_{10, I_{1}} \boldsymbol{U}_{9} \boldsymbol{U}_{6, I_{1}} \boldsymbol{U}_{5} \boldsymbol{U}_{2, I_{1}} \boldsymbol{U}_{1}, \boldsymbol{T}_{5-17}=\boldsymbol{U}_{13} \boldsymbol{U}_{10, I_{1}} \boldsymbol{U}_{9} \boldsymbol{U}_{6, I_{1}} \boldsymbol{U}_{5} \boldsymbol{U}_{2, I_{2}} \boldsymbol{U}_{3} \boldsymbol{U}_{4}, \\
& \boldsymbol{T}_{10-17}=\boldsymbol{U}_{13} \boldsymbol{U}_{10, I_{1}} \boldsymbol{U}_{9} \boldsymbol{U}_{6, I_{2}} \boldsymbol{U}_{7} \boldsymbol{U}_{8}, \boldsymbol{T}_{15-17}=\boldsymbol{U}_{13} \boldsymbol{U}_{10, I_{2}} \boldsymbol{U}_{11} \boldsymbol{U}_{12} .
\end{aligned}
$$

In Eq. (2), the subscript $j-1$ denotes the transfer direction from the component $j$ to the root component 17. $\boldsymbol{U}_{1}, \boldsymbol{U}_{5}, \boldsymbol{U}_{9}$ and $\boldsymbol{U}_{13}$ are the transfer matrices of beam component $\boldsymbol{U}^{\text {Beam }} \cdot \boldsymbol{U}_{3}, \boldsymbol{U}_{7}$ and $\boldsymbol{U}_{11}$ are the transfer matrices of linear translational spring component $\boldsymbol{U}^{\text {spring }} \cdot \boldsymbol{U}_{4}, \boldsymbol{U}_{8}$ and $\boldsymbol{U}_{12}$ are the transfer matrices of lumped mass component $\boldsymbol{U}^{\text {mass }} \cdot \boldsymbol{U}_{2, I_{1}}, \boldsymbol{U}_{2, I_{2}}, \boldsymbol{U}_{6, I_{1}}, \boldsymbol{U}_{6, I_{2}}, \boldsymbol{U}_{10, I_{1}}$ and $\boldsymbol{U}_{10, I_{2}}$ are the transfer matrices of massless rigid bodies 2,6 and 10, respectively, with multi-input and single output, as follows [6]; $\boldsymbol{U}_{4, I_{1}}=\boldsymbol{U}_{6, I_{1}}=\boldsymbol{U}_{10, I_{1}}=\left[\begin{array}{cccc}1 & 0 & 0 & 0 \\ 0 & 1 & 0 & 0 \\ 0 & 0 & 1 & 0 \\ 0 & 0 & 0 & 1\end{array}\right], \quad \boldsymbol{U}_{4, I_{2}}=\boldsymbol{U}_{6, I_{2}}=\boldsymbol{U}_{10, I_{2}}=\left[\begin{array}{ll}0 & 0 \\ 0 & 0 \\ 0 & 0 \\ 0 & 1\end{array}\right]$. The main transfer equation does not describe the geometrical relationship between different input ends and the number of unknown variables is more than that of algebraic equations. Therefore, another set of equations named geometrical equations (GEs) should be introduced, which describes the geometrical relationship between the first input and $(r=2,3,4, \ldots)$ input end of the component. GEs can be written in the following form $\boldsymbol{H}_{j, I_{1}} \boldsymbol{Z}_{j, I_{1}}=\boldsymbol{H}_{j, I_{r}} \boldsymbol{Z}_{j, I_{r}}$. The GEs corresponding to the dummy rigid bodies components 2,6 and 10 can be deduced as follows, respectively

$$
\begin{aligned}
& \boldsymbol{G}_{1-2} \boldsymbol{Z}_{1}+\boldsymbol{G}_{5-2} \boldsymbol{Z}_{5}=\boldsymbol{0} . \\
& \boldsymbol{G}_{1-2}=-\boldsymbol{H}_{2, I_{1}} \boldsymbol{U}_{1}, \quad \boldsymbol{G}_{5-2}=\boldsymbol{H}_{2, I_{2}} \boldsymbol{U}_{3} \boldsymbol{U}_{4} \\
& \boldsymbol{G}_{1-6} \boldsymbol{Z}_{1}+\boldsymbol{G}_{5-6} \boldsymbol{Z}_{5}+\boldsymbol{G}_{10-6} \boldsymbol{Z}_{10}=\boldsymbol{0} . \\
& \boldsymbol{G}_{1-6}=-\boldsymbol{H}_{6, I_{1}} \boldsymbol{U}_{5} \boldsymbol{U}_{2, I_{1}} \boldsymbol{U}_{1}, \boldsymbol{G}_{5-6}=-\boldsymbol{H}_{6, I_{1}} \boldsymbol{U}_{5} \boldsymbol{U}_{2, I_{2}} \boldsymbol{U}_{3} \boldsymbol{U}_{4}, \boldsymbol{G}_{10-6}=\boldsymbol{H}_{6, I_{2}} \boldsymbol{U}_{7} \boldsymbol{U}_{8} \\
& \boldsymbol{G}_{1-10} \boldsymbol{Z}_{1}+\boldsymbol{G}_{5-10} \boldsymbol{Z}_{5}+\boldsymbol{G}_{10-10} \boldsymbol{Z}_{10}+\boldsymbol{G}_{10-15} \boldsymbol{Z}_{15}=\boldsymbol{0} . \\
& \boldsymbol{G}_{1-10}=-\boldsymbol{H}_{10, I_{1}} \boldsymbol{U}_{9} \boldsymbol{U}_{6, I_{1}} \boldsymbol{U}_{5} \boldsymbol{U}_{2, I_{1}} \boldsymbol{U}_{1}, \boldsymbol{G}_{5-10}=-\boldsymbol{H}_{10, I_{1}} \boldsymbol{U}_{9} \boldsymbol{U}_{6, I_{1}} \boldsymbol{U}_{5} \boldsymbol{U}_{2, I_{2}} \boldsymbol{U}_{3} \boldsymbol{U}_{4}, \\
& \boldsymbol{G}_{10-10}=-\boldsymbol{H}_{10, I_{1}} \boldsymbol{U}_{9} \boldsymbol{U}_{6, I_{2}} \boldsymbol{U}_{7} \boldsymbol{U}_{8}, \boldsymbol{G}_{10-15}=\boldsymbol{H}_{10, I_{2}} \boldsymbol{U}_{11} \boldsymbol{U}_{12}
\end{aligned}
$$

In Eq. (3), $\boldsymbol{H}_{2, I_{1}}=\boldsymbol{H}_{6, I_{1}}=\boldsymbol{H}_{10, I_{1}}=\left[\begin{array}{llll}1 & 0 & 0 & 0\end{array}\right]$, and $\boldsymbol{H}_{2, I_{2}}=\boldsymbol{H}_{6, I_{2}}=\boldsymbol{H}_{10, I_{2}}=\left[\begin{array}{ll}1 & 0\end{array}\right]$ [6]. To the end, by combining Eqs. (2, and 3), the overall transfer equation of the entire system shown in Fig. 1(b) can be obtained

$$
\left.\left.\boldsymbol{U}_{\text {all }}\right|_{7 \times 14} \boldsymbol{Z}_{\text {all }}\right|_{14 \times 1}=\boldsymbol{0} \text {. where }\left.\quad \boldsymbol{U}_{\text {all }}\right|_{7 \times 14}=\left[\begin{array}{ccccc}
\boldsymbol{T}_{1-17}^{4 \times 4} & \boldsymbol{T}_{5-17}^{4 \times 2} & \boldsymbol{T}_{10-17}^{4 \times 2} & \boldsymbol{T}_{15-17}^{4 \times 2} & -\boldsymbol{I}^{4 \times 4} \\
\boldsymbol{G}_{1-2}^{1 \times 4} & \boldsymbol{G}_{5-2}^{1 \times 2} & \boldsymbol{0}^{1 \times 2} & \boldsymbol{0}^{1 \times 2} & \boldsymbol{0}^{1 \times 4} \\
\boldsymbol{G}_{1-6}^{1 \times 4} & \boldsymbol{G}_{5-6}^{1 \times 2} & \boldsymbol{G}_{10-6}^{1 \times 2} & \boldsymbol{0}^{1 \times 2} & \boldsymbol{0}^{1 \times 4} \\
\boldsymbol{G}_{1-10}^{1 \times 4} & \boldsymbol{G}_{5-10}^{1 \times 2} & \boldsymbol{G}_{10-10}^{1 \times 2} & \boldsymbol{G}_{10-15}^{1 \times 2} & \boldsymbol{0}^{1 \times 4}
\end{array}\right],\left.\quad \boldsymbol{Z}_{\text {all }}\right|_{14 \times 1}=\left[\begin{array}{c}
\boldsymbol{Z}_{1}^{4 \times 1} \\
\boldsymbol{Z}_{5}^{2 \times 1} \\
\boldsymbol{Z}_{10}^{2 \times 1} \\
\boldsymbol{Z}_{15}^{2 \times 1} \\
\boldsymbol{Z}_{17}^{4 \times 1}
\end{array}\right] .
$$

At the boundaries, half of these SVs are zero due to known constraints, which are at the four tips $\boldsymbol{Z}_{1}=\left[\begin{array}{llll}0 & 0 & M_{z} & Q_{y}\end{array}\right]^{T}, \boldsymbol{Z}_{5}=\left[\begin{array}{ll}Y & 0\end{array}\right]^{T}, \quad \boldsymbol{Z}_{10}=\left[\begin{array}{ll}Y & 0\end{array}\right]^{T}, \quad \boldsymbol{Z}_{15}=\left[\begin{array}{ll}Y & 0\end{array}\right]^{T} \quad$ and $\quad$ at the 
(a)

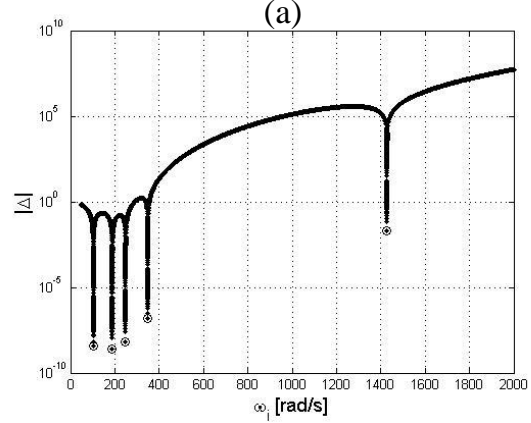

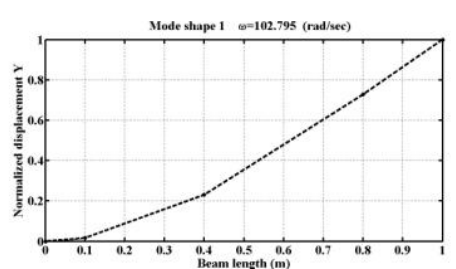

(b)

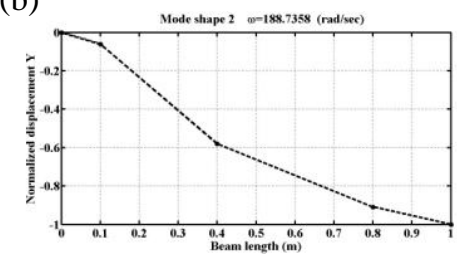

Figure 2. (a) Root scanning of a specific frequency band for the cantilever beam carrying three spring-mass systems shown in Fig. 1, and (b) The corresponding first two mode shapes

$\operatorname{root} \boldsymbol{Z}_{17}=\left[\begin{array}{llll}Y & \Theta_{z} & 0 & 0\end{array}\right]^{T}$. Thus, Eq. (4) reduces to $\overline{\boldsymbol{U}}_{\text {all }} \overline{\boldsymbol{Z}}_{\text {all }}=\boldsymbol{0}$ where $\overline{\boldsymbol{Z}}_{\text {all }}$ is composed of the unknown elements only and $\overline{\boldsymbol{U}}_{\text {all }}$ is a square matrix resulting from elimination of all columns of $\boldsymbol{U}_{\text {all }}$ associated with zeros in $\boldsymbol{Z}_{\text {all }}$. Finally, $\overline{\boldsymbol{U}}_{\text {all }}$ is only a highly nonlinear algebraic function of the unknown natural frequency. For non-trivial solutions, the characteristic equation $\Delta(\omega)=\operatorname{det} \overline{\boldsymbol{U}}_{\text {all }} \stackrel{!}{=} 0$ has to be fulfilled. Recursive eigenvalue search algorithm [9] is employed to determine the system frequencies. Once the required band of the natural frequencies is determined, one may generate the corresponding free vibration mode shapes using Singular Value Decomposition (SVD) [10]. SVD is based on a theorem from linear algebra, which says that a rectangular $m \times n$ matrix $\overline{\boldsymbol{U}}_{\text {all }}$ can be broken down into the product of three matrices; an orthogonal $m \times m$ matrix $\overline{\boldsymbol{U}}_{m \times m}$, a $m \times n$ diagonal matrix $\overline{\boldsymbol{D}}_{m \times n}$, and the transpose of a $n \times n$ orthogonal matrix $\overline{\boldsymbol{V}}_{n \times n}^{\mathrm{T}}$

$$
\overline{\boldsymbol{U}}_{\text {all } m x n}=\overline{\boldsymbol{U}}_{m x m} \overline{\boldsymbol{D}}_{m x n} \overline{\boldsymbol{V}}_{n x n}^{T} .
$$

where $\overline{\boldsymbol{U}}^{T} \overline{\boldsymbol{U}}=\boldsymbol{I} ; \overline{\boldsymbol{V}}^{T} \overline{\boldsymbol{V}}=\boldsymbol{I}$. The columns of $\overline{\boldsymbol{U}}$ are orthogonal eigenvectors of $\overline{\boldsymbol{U}}_{\text {all }} \overline{\boldsymbol{U}}_{\text {all }}^{T}$, the columns of $\overline{\boldsymbol{V}}$ are orthonormal eigenvectors of $\overline{\boldsymbol{U}}_{\text {all }}^{T} \overline{\boldsymbol{U}}_{\text {all }}$, and $\overline{\boldsymbol{D}}$ is a diagonal matrix containing the square roots of eigenvalues from $\bar{U}$ or $\bar{V}$ in descending order. Substitution of Eq. (5) in $\overline{\boldsymbol{U}}_{\text {all }}(\omega) \overline{\boldsymbol{Z}}_{\text {all }}=\boldsymbol{0}$, yields $\bar{U} \bar{D} \bar{V}^{T} \bar{Z}_{\text {all }}=\boldsymbol{0}$.

\section{Results and Discussions}

The reliability of the theoretical work using MSTMM with tree scenario, recursive scanning algorithm in the context of free vibration and the Matlab computer program developed for this paper are confirmed by comparing the present result with those obtained from the example of Table 2 in Ref. [5] as shown in Fig. 1. In Ref. [5], the natural frequencies and the corresponding mode shapes of a uniform cantilever beam carrying "any number of" elastically mounted point masses are determined by means of the analytical-and-numerical-combined method (ANCM). The cantilever beam have the following geometrical and material properties $L=1 \mathrm{~m}, d=0.05 \mathrm{~m}, E=2.069 \times 10^{11} \mathrm{~N} / \mathrm{m}^{2}, \bar{m}=15.3875 \mathrm{~kg} / \mathrm{m}$ and $\rho=7.8367 \times 10^{3} \mathrm{~kg} / \mathrm{m}^{3}$. The positions of the three spring masses along the beam are $x_{1}=0.1 L, x_{2}=0.4 L$ and $x_{3}=0.8 L$, respectively. The spring constants of these three spring mass systems are $K_{y_{1}}=3 \times E I / L^{3}, K_{y_{2}}=4.5 \times E I / L^{3}, K_{y_{3}}=6 \times E I / L^{3}$ and the magnitude of the three masses are $M_{1}=0.2 \times \bar{m} \times L, M_{2}=0.5 \times \bar{m} \times L, M_{3}=1.0 \times \bar{m} \times L \quad$ [5]. Applying fMin1D algorithm $\left([50,2000], N_{x 0}=2000, \varepsilon=10^{-8}\right)[9]$, the absolute value of the determinant is shown in Fig. 2(a). The first five natural frequency values of the entire system are presented in Table 1. Summarizing, MSTMM results are in good agreement with Ref. [5] and it's able to handle this kind of problems. The mode shape of each computed natural frequency $\omega_{i}$ are evaluated following the solutions of $\overline{\mathbf{Z}}_{\text {all }}$ which are given by the last column of $\bar{V}$ corresponding to zero singular values. The corresponding first two mode shapes $Y$ of the normalized displacement are shown in Fig. 2(b). 
Table 1. The lowest five natural frequencies for cantilever beam carrying three spring-mass systems

\begin{tabular}{|c|c|c|c|c|c|c|}
\hline \multirow[t]{2}{*}{ Method } & \multicolumn{5}{|c|}{ Natural frequency $(\mathrm{rad} / \mathrm{sec})$} & \multirow{2}{*}{$\begin{array}{c}\mathrm{CPU} \\
\text { time } \\
(\mathrm{sec})\end{array}$} \\
\hline & $\omega_{1}$ & $\omega_{2}$ & $\omega_{3}$ & $\omega_{4}$ & $\omega_{5}$ & \\
\hline FEM [5] & 102.79 & 188.73 & 248.66 & 349.11 & 1428.03 & 19.18 \\
\hline ANCM [5] & 102.71 & 188.76 & 248.51 & 349.14 & 1427.97 & 5.05 \\
\hline MSTMM & 102.79 & 188.73 & 248.64 & 349.11 & 1427.96 & 2.25 \\
\hline
\end{tabular}

\section{Summary}

Starting from the principle of mechanics and the elementary formulations for the flexible beam, the free vibration analysis of transversely vibrating system made up of an Euler-Bernoulli beam to which spring/mass systems are attached across the span is performed using Transfer Matrix Method of Linear Multibody Systems (MSTMM) scenario. Although the number of coupling spring/mass systems considered in the example given was limited to three, there is no inherent difficulty in extending the current method to solve the problems of vibration of systems consisting of any number of spring/mass/dashpot systems. The numerical result obtained reveals that the eigenfrequencies calculated by this method is in very good agreement with those obtained by the published literature.

\section{Acknowledgment}

The authors gratefully acknowledge the financial support of this research from the Natural Science Foundation of China under number 11472135 and International Cooperation and Exchange Department in Nanjing University of Science and Technology.

\section{References}

[1] L. A. Parnell, M. H. Cobble, Lateral displacement of a vibrating cantilever beam with a concentrated mass. Journal of Sound and Vibration, 44 (2), pp. 499-511, 1976.

[2] P. A. A. Laura, E.A. Susemihl, J.L. Pompo, L.E. Luisoni, R. Gelos, On the dynamic behaviour of structural elements carrying elastically mounted concentrated masses. Applied Acoustics, 10 (2), pp. 121-145, 1977.

[3] H. Larrondo, D. Avalos, P. A. A. Laura, Natural frequencies of a Bernoulli beam carrying an elastically mounted concentrated mass. Ocean Engineering, 19 (5), pp. 461-468, 1992.

[4] M. U. Jen, E. B. Magrab, Natural frequencies and mode shapes of beams carrying a two-degree-of-freedom spring-mass system. Journal of Vibration and Acoustics, 115 (2), pp. 202-209, 1993.

[5] J. S. Wu, H. M. Chou, Free vibration analysis of a cantilever beam carrying any number of elastically mounted point masses with the analytical-and-numerical-combined method. Journal of Sound and Vibration, 213 (2), pp. 317-332, 1998.

[6] Laith K. Abbas, Q. B. Zhou, H. Hendy, Xiaoting Rui, Transfer matrix method for determination of the natural vibration characteristics of elastically coupled launch vehicle boosters. Acta Mechanica Sinica, 31 (4), pp. 570-580, 2015.

[7] Xiaoting Rui, L. Yun, Y. Lu, B. He, G. P. Wang, Transfer Matrix Method of Multibody System and its Application. Science Press, Beijing, 2008 (in Chinese).

[8] Xiaoting Rui, J. S. Zhang, Q. B. Zhou, Automatic deduction theorem of overall transfer equation of multibody system. Advances in Mechanical Engineering, 6, Article ID 378047, 2014.

[9] D. Bestle, Laith K. Abbas, Xiaoting Rui, Recursive eigenvalue search algorithm for transfer matrix method of linear flexible multibody systems. Multibody System Dynamics, 32 (4), pp. 429-444, 2014.

[10] Laith K. Abbas, Li M J, Rui Xiaoting, Transfer matrix method for the determination of the natural vibration characteristics of realistic thrusting launch vehicle-Part I. Mathematical Problems in Engineering, 2013; Article ID 764673, 16 pages, 2013. 